\title{
AVALIAÇÃO DO PERFIL IMUNOGÊNICO DE VACINAS INATIVADAS CONTRA AGALAXIA CONTAGIOSA EM CAPRINOS
}

\author{
(Evaluation of the immunogenic profile of inactivated vaccines against contagious \\ agalactia in goats)
}

Aline Antas Cordeiro Cavalcanti' ${ }^{1}$ Ana Claudia Campos², Marcia Almeida Melo', Aline Guedes Mamede Moraes ${ }^{1}$, Aldenir Cavalcanti Lima', Edisio Oliveira Azevedo².

${ }^{1}$ Universidade Federal de Campina Grande, Campina Grande, PB, Brasil; 2Universidade Federal de Sergipe, Aracaju, SE, Brasil.

1'Autor para correspondência: aline.antas@hotmail.com

RESUMO: Este trabalho teve como objetivo avaliar o perfil imunogênico em caprinos de três vacinas inativadas contra agalaxia contagiosa $(A C)$, preparadas com linhagens de Mycoplasma agalactiae isoladas no Brasil. A vacina 1 foi adsorvida em hidróxido de alumínio, a vacina 2 em Montanide IMS-2215-VG e a vacina 3 em Montanide Gel-01. O perfil proteico do antígeno vacinal foi avaliado por SDS-PAGE e a imunogenicidade da vacina pela técnica de Western blotting. A vacina foi administrada em três doses, com intervalo de 21 dias entre a primeira e a segunda e de 180 dias entre a segunda e a terceira. O pool de soros de dez caprinos coletados nos períodos $0,21,35,90,150,210$, 270 e 360 dias pós-vacinação foram testados pela técnica de Western blotting. A vacina 2 foi mais antigênica, seguida da vacina 1. Os anticorpos foram observados 21 dias após a primeira dose para as três vacinas. Houve diferença no perfil das proteínas que foram reconhecidas por anticorpos para as vacinas 1 e 2; a vacina 1 estimulou a produção de anticorpos contra as proteínas P48 e P55 e a vacina 2 contra a P48 e a P80. Conclui-se que as vacinas 1 e 2 tiveram um perfil semelhante quanto a indução da produção de anticorpos.

Palavras-chave - adjuvante; imunoblotting; Mycoplasma agalactiae; pequeno ruminante.

ABSTRACT - This study aimed to evaluate the immunogenicity in goats of three inactivated vaccines against contagious agalactia (CA), prepared with a Brazilian field strain of Mycoplasma agalactiae. Three different vaccine formulations were evaluated: Vaccine 1 antigen adsorbed onto aluminum hydroxide; Vaccine 2 - antigen adsorbed onto Montanide IMS-VG-2215, and Vaccine 3 - antigen adsorbed onto Montanide Gel-01. The protein profile of the vaccine antigen was evaluated by SDS-PAGE and vaccine immunogenicity by Western blotting technique. The vaccines were administered in three doses, with an interval of 21 days between the first and second doses and 180 days between the second and the third doses. Sera pool collected from ten goats in periods 0, 21, 35, 90, 150, 210, 270 and 360 days after first dose were tested by Western blotting technique. Vaccine 2 was more antigenic than vaccine 1 . The antibodies were observed 21 days after the first dose for all vaccines. It has difference in the proteins profile that were recognized by antibodies to vaccines 1 and 2: vaccine 1 stimulated antibodies production against P48 and P55 proteins and the vaccine 2 against P80 and P48 proteins. We concluded that vaccines 1 and 2 had a similar profile in induction of antibody production.

Palavras-chave - adjuvant; immunoblotting; Mycoplasma agalactiae; small ruminant. 


\section{INTRODUÇ̃̃O}

O Mycoplasma agalactiae é um dos principais agentes causadores da agalaxia contagiosa (AC) de caprinos e ovinos, que é uma síndrome caracterizada por agalaxia, poliartrite e ceratoconjuntivite. A infecção se dissemina rapidamente no rebanho, determinando grandes perdas econômicas, decorrentes da diminuição da produção do leite e da morte dos animais. A enfermidade é endêmica em países do Mediterrâneo, África e região oeste e central da Ásia e é uma doença emergente em países da América e no Japão (Azevedo et al., 2006; OIE, 2012).

Uma das estratégias de controle da AC é a adoção da antibioticoterapia por longos períodos que reduz os sinais clínicos, mas mantém um grande número de animais portadores. Além desta, a indução de partos com separação imediata das crias e o uso de bioterápico foram estudadas (Alcântara et al., 2003; Marinho, 2008).

As primeiras vacinas produzidas contra AC em caprinos e ovinos datam da década de 70 (Foggie et al., 1970), mas apenas na década de 90 é que passou a ser utilizada mais intensamente na Europa, onde a doença causa perdas econômicas consideráveis (León Vizcaíno et al., 1995).

No Brasil, foram testadas vacinas inativadas de $M$. agalactiae adsorvidas com diferentes adjuvantes (hidróxido de alumínio, Montanide IMS-2215-VG e Montanide Gel-01), obtendo-se bons resultados na que continha adjuvante oleoso (Campos, 2012).

O $M$. agalactiae pode alterar os antígenos de superfície de acordo com $\mathrm{De} \mathrm{La} \mathrm{Fe}$ et al. (2006). Em amostras brasileiras de $M$. agalactiae, isoladas de leite caprino de infecção natural, foram identificadas proteínas com peso molecular que variaram de 30 a $135 \mathrm{kD}$ no SDS-PAGE, com imunogenicidade para as que possuíam pesos moleculares de 135, 55 e 48 kD (Campos, 2012).

A imunização como estratégia de prevenção da agalaxia contagiosa, pode se tornar uma realidade no Brasil, pois os primeiros estudos demonstraram 0 potencial das vacinas produzidas com amostras isoladas no Nordeste do país. Nesse sentido, o objetivo deste trabalho foi avaliar a produção de anticorpos antiproteínas $M$. agalactiae em caprinos vacinados com antígeno bruto inativado, adsorvido com diferentes adjuvantes.

\section{MATERIAL E MÉTODOS}

As vacinas foram preparadas a partir da amostra de $M$. agalactiae (BrPB3.03) previamente caracterizada (Campos, 2012), cultivada durante 96 horas em caldo Hayflick modificado, $\mathrm{pH}$ 7,8 , enriquecido com $20 \%$ de soro equino inativado a $37^{\circ} \mathrm{C}$ em microaerofilia. $O$ cultivo foi centrifugado duas vezes a $3800 \mathrm{~g}$ durante uma hora e ressuspenso em tampão salino fosfato [PBS, $137 \mathrm{mM} \mathrm{NaCl}, 2,7 \mathrm{mM} \mathrm{KCl}, 10,2$ $\left.\mathrm{mM} \mathrm{Na}_{2} \mathrm{HPO}_{4}, 1,8 \mathrm{mM} \mathrm{KH}_{2} \mathrm{PO}_{4}, \mathrm{pH} 7,6\right]$.

A suspensão bacteriana obtida foi ajustada para uma concentração proteica pré-inativação de $5 \mathrm{mg} /$ dose da vacina e inativadas com formaldeído a $0.4 \%$ (v/v) por 24 horas a $37^{\circ} \mathrm{C}$. A suspensão inativada adsorvida sob agitação mecânica e constante durante três horas a temperatura ambiente, com o respectivo adjuvante: a) Vacina 1: Gel de hidróxido de alumínio na concentração de $6 \mathrm{mg} / \mathrm{mL}$ de vacina (adjuvante aquoso); b) Vacina 2: Montanide IMS 2215 VG PR (Seppic Inc.), na concentração de $25 \%$ (v/v) (adjuvante oleoso); c) Vacina 3: Montanide Gel 01 PR (Seppic Inc.), a $5 \%(\mathrm{v} / \mathrm{v})$ (adjuvante aquoso), descrito por Campos (2012). As vacinas foram estocadas a $4 \stackrel{\circ}{ } \mathrm{C}$.

Quarenta caprinos sem padrão racial definido, machos e fêmeas, com idade variando de 6 a 48 meses, foram distribuídos aleatoriamente em três 
grupos vacinais e um controle, com dez animais cada. A imunização foi realizada por via subcutânea na região da axila direita e esquerda, previamente tricotomizada, com duas doses de $2 \mathrm{~mL}$ com intervalo de 21 dias. Uma terceira dose foi administrada 180 dias após a segunda vacinação. O grupo controle recebeu o mesmo protocolo de vacinação utilizando PBS estéril como inoculo, como descrito por Campos (2012).

Os soros dos caprinos foram coletados nos dias $0,21,35,90,150$, 210,270 e 360 dias após a 11 vacinação.

Para análise do perfil protéico pelo Western blotting (WB), a amostra de $M$. agalactiae (BrPB3.03) em caldo Hayflick modificado $(1,5 \mathrm{~mL})$ foi centrifugada a $12000 \mathrm{~g}$ por 20 minutos e o pellet foi ressuspenso em PBS, $\mathrm{pH}$ 7,6. A concentração de proteína foi determinada por espectrofotometria (BioPhotometer Plus, Eppendorf, Alemanha) utilizando um filtro de 280. A amostra foi misturada em tampão de lise [500 mM Tris/HCl pH 6,8, 4,6\% (p/v) SDS, $20 \%$ (v/v) glicerol, $10 \%$ (v/v) 2mercaptoetanol e $0,004 \%$ azul de bromofenol] e fervida por $5 \mathrm{~min}$. Posteriormente, $15 \mu \mathrm{g}$ de proteínas de M. agalactiae foram separadas por SDS-PAGE 12\% (Laemmli, 1970). A eletroforese foi realizada a $250 \mathrm{~V}$ e 50 $\mathrm{mA}$ por gel durante 40 minutos, com posterior coloração do gel com a solução de Comassie blue [0,005\% (p/v) azul brilhante de Coomassie R-250, 7\% (v/v) ácido acético, 25\% (v/v) metanol].

As proteínas separadas por SDSPAGE foram transferidas para membrana de nitrocelulose $0,45 \mu \mathrm{m}$ a 8 $\mathrm{V}$ e $700 \mathrm{~mA}$ por 70 minutos. As membranas foram bloqueadas em solução de bloqueio [PBS pH 7,6; $2 \%$ $(\mathrm{p} / \mathrm{v})$ leite em pó desnatado (LPD)] por 30 minutos a $37^{\circ} \mathrm{C}$, e em seguida lavadas três vezes em PBS pH 7,6 por 5 minutos.

A membrana sensibilizada foi cortada em tiras e imersa no pool dos soros dos caprinos vacinados diluído 1:100 e nos controles positivo e negativo diluídos 1:200 em solução de bloqueio e incubadas a $37^{\circ} \mathrm{C}$ por 45 minutos. O controle negativo do WB constituiu de um pool de soros de cinco caprinos não imunizados e negativos para $M$. agalactiae e o controle positivo de um pool de soros de cinco animais com sinais clínicos de AC, testados previamente pelo ELISA indireto (Campos et al., 2009).

Após incubação, as tiras foram lavadas três vezes por 5 minutos a temperatura ambiente com PBS e em seguida incubadas com conjugado proteína $\mathrm{G}$ peroxidase (Sigma-Aldrich) diluído 1:9000. As tiras foram novamente submetidas a lavagem e incubadas em solução de revelação $[0,1$ M tampão citrato $\mathrm{pH} \mathrm{5,0;0,3} \mathrm{mg/mL} \mathrm{3'-}$ 3'-5'-5'-tetrametilbenzidina (TMB) e $0,38 \%$ peróxido de hidrogênio]. Após cinco minutos, a reação foi interrompida com água destilada.

\section{RESULTADOS E DISCUSSÃO}

A análise da linhagem de Mycoplasma agalactiae utilizada na vacina pela eletroforese em gel de poliacrilamida (SDS-PAGE) mostra bandas proteicas de $20 \mathrm{kDa}$ a $120 \mathrm{kDa}$. Três bandas localizadas entre 20 e 30 kDa, 50 e 70 kDa e acima de 120 kDa não tiveram o peso molecular definido a partir do peso molecular padrão utilizado neste estudo (Fig. 1). Resultados de De $\mathrm{La} \mathrm{Fe}$ et al. (2006) mostram várias proteínas entre os intervalos anteriormente citados e em torno de 5 bandas com pesos moleculares acima de $102 \mathrm{kDa}$ e relatam a imunogenicidade de proteínas com pesos moleculares de 24-25, 60 e 104$105 \mathrm{kDa}$. 
PM AMOSTRA VACINALDEM. agalactiae

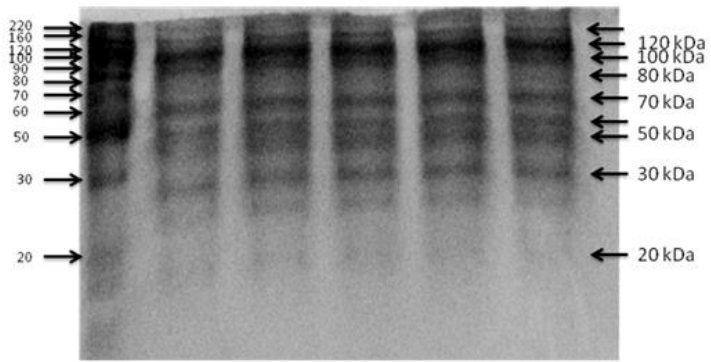

Figura 1 - SDS-PAGE de proteína total de $M$. agalactiae corado com Comassie blue. Numeração à esquerda: peso molecular; Numeração à direita: pesos moleculares das proteínas de $M$. agalactiae.

Através do imunoblotting foi possível verificar a presença de anticorpos IgG anti- $M$. agalactiae no pool dos soros dos caprinos vacinados com as três vacinas, entretanto, as vacinas 2 (adjuvante oleoso) foi mais antigênica, seguida da vacina 1 (adjuvante hidróxido de alumínio), confirmando os achados de Campos (2012) através do ELISA indireto. Adicionalmente, também houve diferença no perfil das proteínas que foram reconhecidas por anticorpos para as vacinas 1 e 2; a vacina 1 estimulou a produção de anticorpos contra as proteínas P48 e P55 e a vacina 2 contra a $\mathrm{P} 48$ e a $\mathrm{P} 80$.

Buonavoglia et al. (1998) e Greco et al. (2002) observaram que a vacina associada ao adjuvante oleoso foi mais imunogênica do que a vacina com adjuvante hidróxido de alumínio. Buonavoglia et al. (2008) verificaram que a vacina constituída por uma emulsão de três óleos minerais impediu a infecção dos animais, caracterizada por sinais clínicos e eliminação da bactéria pelo leite e por via nasal, após o desafio.

Em relação à presença de anticorpos anti- $M$. agalactiae, observouse que já havia produção de anticorpos 21 dias após a primeira dose para as três vacinas. Nos períodos 90, 150 e 210 houve uma queda na quantidade de anticorpos (Fig. 2), representado pela diminuição da intensidade das bandas no Western blotting (Fig. 2). A diminuição na produção de anticorpos em um curto período pós-vacinação, provavelmente, deve-se ao fato de que a vacina inativada estimular níveis de anticorpos mais baixos e menos persistentes, necessitando de repetições das doses em períodos mais curtos (León Vizcaíno et al., 1995; Buonavoglia et al., 1998); fato comprovado com o aumento da intensidade das bandas nos dias 280 e 360 após o reforço vacinal administrado 180 dias após a segunda dose (Fig. 2).

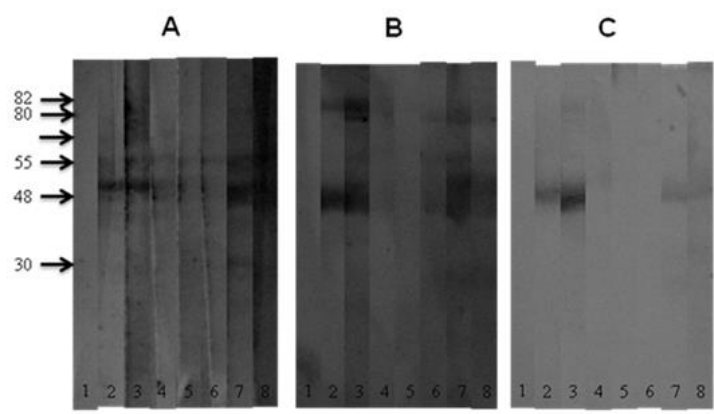

Figura 2 - Imunoblotting de proteína total de $M$. agalactiae utilizando soros de caprinos vacinados com as vacinas experimentais 1 (A), 2 (B) e 3 (C). A numeração na fita corresponde aos dias após a $1^{\text {a }}$ dose da vacinação: $1: 1^{\text {a }}$ dose da vacina; 2: 21 dias pós-vacinação; $3: 35$ dias pósvacinação; 4: 90 dias pós-vacinação; 5: 150 dias pósvacinação; 6: 210 dias pós-vacinação; 7: 270 dias pósvacinação e 8: 360 dias pós-vacinação. Os pesos moleculares das proteínas em $\mathrm{kDa}$ estão indicados à esquerda.

Pelo Western blotting, observa-se que, após as duas primeiras doses, a vacina constituída de extrato total de $M$. agalactiae foi capaz de estimular a produção de anticorpos contra as proteínas com $82,80,55,48$. Uma banda tênue com peso molecular de 30 kDa é vista nas vacinas 1 e 2 após a terceira dose. Após a terceira dose, ministrada 180 dias após a segunda, as proteínas mais antigênicas foram as de 80,55 e 48 kDa. Anticorpos antiproteínas com 55 e 48 kDa persistiram mesmo no período que houve queda do título. Após a terceira dose vacinal reapareceram as bandas de 55 e 48 $\mathrm{kDa}$ para as vacinas 1 e 2, $80 \mathrm{kDa}$ para a vacina 2 e $48 \mathrm{kDa}$ para a vacina 3 (Fig. 2). 
Campos (2012) relatou que a proteína P48 foi a mais imunogênica em soros de caprinos naturalmente infectados. A P48 é uma lipoproteína que possui uma potente ação imunoestimulante, sendo um dos principais antígenos encontrados na infecção por M. agalactiae (Rosati et al., 1999; 2000). Chessa et al. (2009) imunizaram camundongos com uma vacina de DNA contendo o gene da P48 e houve estímulo da resposta imune celular (Th1) e humoral (Th2); IgG1 antiP48 recombinante foi identificado por ELISA.

Anticorpos anti-P55 foram observados 21 dias após a primeira vacinação, e estavam consideravelmente diminuídos após 90 dias, nas vacinas 2 e 3; reaparecendo após a dose de reforço nas vacinas 2 e 3. Santona et al. (2002) e Tola et al. (1997) relatam que a P55 é intensamente imunogênica em animais infectados com $M$. agalactiae.

Anticorpos IgG anti- a fração de 80 kDa foram observados 35 dias após a primeira vacinação, além de uma outra banda de aproximadamente $82 \mathrm{kDa}$ na vacina 2. No artigo de Tola et al. (1997) observa-se uma banda imunogênica acima da de $80 \mathrm{kDa}$ revelada com soros de ovinos com 30 dias após o início da sintomatologia clínica, mas os autores não tecem comentários.

A vacinação com o extrato bruto também foi capaz de estimular a resposta humoral contra a $\mathrm{P} 80$, uma lipoproteína de membrana (Tola et al., 2001), que foi reconhecida por soros de ovinos em início da sintomatologia clínica (Tola et al., 1997), sugerindo-a como um marcador para diagnóstico precoce.

Para as vacinas 1 e 2 , percebe-se uma discreta banda imunogênica de aproximadamente $30 \mathrm{kD}$ no immunoblotting realizado aos 270 e 360 dias, que corresponde 70 dias após a terceira aplicação. De la Fe et al. (2006) e Tola et al. (1997) observaram que esta proteína também é imunogênica na infecção natural em ovinos. Fleury et al. (2001) observaram que a P30 estimulou uma resposta humoral em ovelhas infectadas artificialmente com diferentes linhagens de $M$. agalactiae; os anticorpos persistiram durante, pelo menos, 2 meses após a infecção. Nas vacinas estudadas, a P30 foi pouco antigênica, que pode ser em função de modificaçãoes na estrutura da proteína pelo formol ou durante a confecção da vacina.

O gene para a P30 é específico para M. agalactiae (Fleury et al., 2001) e a produção de anticorpos anti-P30 pode indicar que a linhagem de M. agalactiae utilizada na vacina faça parte do sorotipo A-D, de acordo com Bergonier et al. (1996).

\section{CONCLUSÃO}

As vacinas 1 e 2 tiveram um perfil semelhante quanto a indução da produção de anticorpos contra proteínas de superfície de Mycoplasma agalactiae.

\section{REFERÊNCIAS}

ALCÂNTARA, M.D. B.; AZEVEDO, E.O.; FARIAS, A.A. et al. Indução de parto e separação das crias para controle da agalaxia contagiosa em caprinos. In: CONG. LATINAMER. BUIATRIA, 11, 2003, Salvador. Anais... Salvador: [s.n.] 2003. p.71.

AZEVEDO, E.O.; ALCÂNTARA, M.D.B.; NASCIMENTO, E.R. et al. Contagious agalactia by Mycoplasma agalactiae in small ruminants in Brazil: first report. Brazilian Journal of Microbiology, v.37, p.576-581, 2006.

BERGONIER, D.; DE SIMONE, F.; RUSSO, P. et al. Variable expression and geographic distribution of Mycoplasma agalactiae surface epitopes 
demonstrated with monoclonal antibodies. FEMS Microbiology Letters, v.143, n.2-3, p.159-65, 1996.

BUONAVOGLIA, D.; FASANELLA, A.; SAGAZIO, $P$. et al. Persistence of antibodies to Mycoplasma agalactiae in vaccinated sheep. New Microbiologica, v.21, n.2, p.209-212, 1998.

BUONAVOGLIA, D.; GRECO, G.; QUARANTA, V. et al. An oil-emulsion vaccine induces full-protection against Mycoplasma agalactiae infection in sheep. New Microbiologica, v.31, p.117-123, 2008.

CAMPOS, A.C.; TELES, J.A.A.; AZEVEDO, E.O. et al. ELISA protein G for the diagnosis of contagious agalactia in small ruminants. Small Ruminant Research, v.84, p.70-75, 2009.

CAMPOS, A.C. Produção e avaliação de vacina contra agalaxia contagiosa. 2012. 75f. Tese (Doutorado em Ciência Veterinária) - Departamento de Medicina Veterinária, Universidade Federal Rural de Pernambuco, Recife, PE.

CHESSA, B.; PITTAU, M.; PURICELLI, $M$. et al. Genetic immunization with the immunodominant antigen P48 of Mycoplasma agalactiae stimulates a mixed adaptive immune response in BALBc mice. Research in Veterinary Science, v.86, p.414-420, 2009.

DE LA FE, C.; ASSUNÇÃO, P.; ROLALES, R.S. et al. Characterization of protein and antigen variability among Mycoplasma mycoides subsp. mycoides (LC) and Mycoplasma agalactiae field strains by SDS-PAGE and immunoblotting. The Veterinary Journal, v.171, p.532-538, 2006.

FLEURY, B.; BERGONIER, D.; BERTHELOT, $X$. et al. Characterization and analysis of a stable serotypeassociated membrane protein (P30) of Mycoplasma agalactiae. Journal of
Clinical Microbiology, v.39, n.8, p.2814-2822, 2001.

FOGGIE, A.; ETHERIDGE, J.R.; ERDAB, O. et al. Contagious agalactia of sheep and goats preliminary studies on vaccines. Journal of Comparative Pathology, v.80, p.345-359, 1970.

GRECO, G.; CORRETE, M.; BUONAVOGLIA, D. et al. Inactivated vaccine induces protection against Mycoplasma agalactiae infection in sheep. New Microbiologica, v.25, n.1, p.17-20, 2002.

LAEMMLI, U.K. Cleavage of structural proteins during the assembly of the head of bacteriophage T4. Nature, v.227, p.680-685, 1970.

LEON VIZCAINO, L.; GARRIDO ABELLAN, F.; CUBERO PABLO, M.J. et al. Immunoprophylaxis of caprine contagious agalactia due to Mycoplasma agalactiae with an inactivated vaccine. Veterinary Record, v.137, n.11, p.266269, 1995.

MARINHO, M.L. Ação terapêutica do bioterápico de Mycoplasma agalactiae em caprinos com agalaxia contagiosa dos ovinos e caprinos. 2008. 118f. Tese (Doutorado em Ciência Veterinária) - Departamento de Medicina Veterinária, Universidade Federal Rural de Pernambuco, Recife, PE.

ORGANIZAÇÃO MUNDIAL DE SAÚDE ANIMAL - OIE. Contagious agalactia. $2012 . \quad$ Disponível em: <http://www.oie.int/wahis_2/public/wahid .php/Diseaseinformation/Diseasetimelin es>. Acesso em: 13/04/2013.

ROSATI, S.; POZZI, S.; ROBINO, P. et al. P48 major surface antigen of Mycoplasma agalactiae is homologous to a malp product of Mycoplasma fermentans and belongs to a selected family of bacterial lipoproteins. Infection and Immunity, v.67, n.11. p. 62136216, 1999. 
ROSATI, S.; ROBINO, P.; FADDA, M. et al. Expression and antigenic characterization of recombinant Mycoplasma agalactiae P48 major surface protein. Veterinary Microbiology, v.71, p.201-210, 2000.

SANTONA, A.; CARTA, F.; FRAGHI, P. et al. Mapping antigenic sites of an immunodominant surface lipoprotein of Mycoplasma agalactiae, AvgC, with the us of synthetic peptides. Infection and Immunity, v.70, n.1, p.171-176, 2002.

TOLA, S.; MANUTA, D.; COCCO, M. et al. Characterization of surface proteins of Mycoplasma agalactiae during natural infection. FEMS Microbiology Letters, v.154, p.355-362, 1997.

TOLA, S.; CROBEDDU, S.; CHESSA, G. et al. Sequence, cloning, expression and characterisation of the $81-\mathrm{kDa}$ surface membrane protein (P80) of Mycoplasma agalactiae. FEMS Microbiology Letters, v.202, p.45-50, 2001. 